\title{
LE RÉCIT MÉDIATIQUE : L'OBJET ET SA MÉTHODE. POUR UNE MISE EN TENSION DYNAMIQUE
}

\section{Frédéric Antoine ${ }^{1}$}

Lors de sa fondation, il y a cinq ans, les intentions sur lesquelles reposait la mise au monde de l'ORM relevaient de l'opérationnalisation d'intuitions scientifiques, inspirées par un recadrage du mode de préhension du récit puisé à la source des travaux de Paul Ricœur.

Il est aujourd'hui incontestable que ces "intuitions" sur lesquelles l'ORM a bâti ses divers travaux se sont affirmées, solidifiées, sédimentées au contact de la réalité empirique, de l'analyse et de la mise en recherche systématique. Tant et si bien qu'il n'est sans doute pas erroné d'avancer -non sans quelques signes d'une modeste fierté- que le mode particulier de saisie de la problématique communicationnelle médiatique revendiqué par cette équipe de recherche ne semble pas seulement considéré avec un certain intérêt par la communauté des chercheurs en information et communication mais y fait réellement l'objet d'une reconnaissance de plus en plus marquée.

Considérant que les modes d'approche disciplinaires quelque fois particulièrement cloisonnés, utilisés jusqu'ici, n'ont peut-être pas répondu de manière aussi parfaite qu'escompté aux interrogations que lui soumettaient les chercheurs, les chercheurs de l'ORM ont estimé

1 Professeur au Département de communication de l'Université catholique de Louvain et responsable de l'unité de récit médiatique.

Recherches en communication, $\mathrm{n}^{\circ} 7,(1997)$. 
que l'étude des mass-media était -et reste en grande partie- en quête de nouveaux paradigmes.

La démarche intellectuelle, la dynamique de questionnement ainsi que les interactions disciplinaires et méthodologiques que l'on cultive à l'ORM se présentent, à leur échelle, comme autant de moyens de fournir un écho positif à ces attentes de la recherche.

Bien sûr, la méthode dont nous nous revendiquons est jeune, et toujours en élaboration. Reposant sur l'empirie, elle s'édifie par essai et erreur au fil du temps de la recherche et des confrontations (inter)disciplinaires. Elle balbutie donc encore et ne peut revendiquer dès aujourd'hui la fourniture clé sur porte d'un canevas incontestable de (com)préhension de la "réalité" médiatique. Le questionnement et les discussions développés ci-avant dans cet ouvrage à propos de la (ou des) "méthode(s)" relative(s) à quelques-unes seulement des phases d'élaboration du récit médiatique témoignent à l'envi de l'état toujours en devenir de la méthodologie de l'observation du récit telle que nous la concevons.

De même, le type de dynamique privilégié ici se doit, comme tout procédé de questionnement scientifique, d'être soumis à la sagacité et à l'appréciation de ses pairs, ce qui lui permet de se parfaire, mais lui offre aussi l'opportunité de borner, de manière restrictive ou extensive, son champ d'action et de mise en opération.

Nous n'avons jamais entendu esquiver ce débat. La diversité d'identité des spécialistes intervenus ci-avant à ce propos le démontre si besoin en était : jeune et encore porteur de soif de savoir, l'ORM cherche avidement à se nourrir de la confrontation de points de vue... même si, au terme de l'échange, chacun ne se rallie pas entièrement aux arguments de l'autre.

Ainsi, l'ORM ne partagera sans doute jamais complètement les modèles définitionnels trop rigoristes ou microscopiques que l'on pourrait assigner à son domaine. On y discutera notamment encore longtemps les problématiques liées au bornage du récit, à sa clôture ou au rapport entre récit, fiction et réalité.

Et il est assurément bon qu'il en soit ainsi. Que le questionnement, sinon le doute, plane constamment au-dessus de nos travaux. Que l'on y conserve par exemple à tout moment en mémoire, à l'instar d'une véritable épée de Damoclès épistémologique, la menace d'un possible reproche de trop sacrifier au culte du "tout récit" ou plus encore du "tout narratif". 


\section{Une recherche dynamique}

Il est en effet incontestable que tout n'est pas récit, et qu'à tendre à une préhension maximalisante de ce terme, on encourt le risque de perdre les moyens de procéder à une identification spécifique de son objet, comme l'affirment les tenants d'une lecture rigide des conditions d'existence du récit médiatique. Néanmoins, l'ORM n'entendra pas se confiner à une préhension trop étriquée de sa sphère d'étude. Parmi les concepts qui ont traversé les travaux qui précèdent dans cet ouvrage, les termes de "circularité", de "boucle mimétique" et de "mise en tension" revêtent à cet égard une importance plus que particulière. Ils manifestent notre volonté existentielle de dépasser l'état d'une mise en œuvre appliquée de cadres théoriques et méthodologiques préétablis, déjà produits, déjà posés. Ils attestent l'intention primale de cet Observatoire d'appréhender son objet et de le traiter dans le contexte d'une dynamique permanente.

Celle-ci induit nécessairement l'idée d'une circulation tant théorique que méthodologique et empirique entre les trois pôles de la boucle mimétique telle que nous l'avons réfigurée, allant des conditions de production du récit (dont il n'était ici pas question) aux réappropriations des contenus narratifs par leurs usagers en passant par l'étape, essentielle mais non unique, de l'introspection dans le récit en tant que tel.

A la lecture des communications scientifiques qui précèdent, on ajoutera que cette préoccupation de circularité implique aussi la mise en œuvre d'un autre cheminement auquel nous devrons être attentifs : celui qui impose à la recherche, conçue dans un cadre dynamique, de prendre à la fois en considération des objets d'étude et d'analyse relevant de niveaux méthodologiques micro, meso et macro, allant de la dissection fine d'unité narratives à l'interprétation macrosociale de la signification du récit en passant par l'étude des récits emboîtés et de leurs procédés d'enchâssement.

Au-delà du fait qu'il reste à l'ORM bien des étapes à parcourir afin de satisfaire aux exigences de cette grille d'analyse tentaculaire, il faut concéder que cultiver comme règle d'or le pari d'une dynamique méthodologique n'est pas une attitude sans risque. Elle remet en effet en cause quelques-unes des frontières disciplinaires encore bien établies derrière lesquelles s'édifient les forteresses de la science. Le choix de la dynamique relève aussi la gageure de reprofiler ces frontières interdisciplinaires. Et il permet aussi d'oser opérer la 
structuration de son propre objet en recourant notamment au déplacement ou à la "réimplantation" de champs théoriques ou méthodologiques voisins ou connexes.

Cette option, parfois, bouscule ou dérange. Et peut poser question. Sous prétexte d'établir de nouveaux modèles, ne sommesnous pas menacés par une contagion (voire la confusion) méthodologique des genres ? Les "greffes" disciplinaires qu'on y réalise parfois sont-elles exemptes de toute éventualité de rejet?

Il est assurément trop tôt pour trancher ces questions. Une méthode en état de mise en œuvre ne peut elle-même qu'expérimenter les limites de ses capacités. Mais elle doit en être consciente.

A ce propos, il peut être fait confiance à l'ORM qui entend faire sienne cette recommandation de Michel de Certeau :

Pour lire et écrire la culture ordinaire, il faut réapprendre des opérations communes et faire de l'analyse une variante de son objet $^{1}$.

Est-il anormal que ce mode de recadrage soit aussi un peu celui de l'ORM, où l'on se revendique tellement des pratiques du braconnage?

N'est-ce pas en partie ce pour quoi l'Observatoire plaide quand il propose d" "aller voir hors du narratif ce qu'il y a de narratif" ou lorsqu'il évoque "la contagion médiatique" ou "la porosité" des récits médiatiques?

\section{(Re)tour du terrain}

Les notions de "circularité" et de "mise en tension" imposent aussi au chercheur de ne pas sacrifier à l'un des pièges qui menacent fréquemment la communauté scientifique : celui du recours aux interprétations théoriques, aux modèles, aux lectures in abstracto. Celles-ci revêtent souvent, pour le chercheur, l'aspect d'une planche de salut à laquelle on s'agrippe, malgré soi, lorsque la houle de l'expérience chahute trop sensiblement les cadres qu'on s'était établis, reléguant au deuxième plan le retour (ou la descente) sur le terrain.

Pour l'ORM, la méthode et a fortiori l'interprétation ne peuvent précéder l'observation. Et celle-ci ne peut, à chaque fois, se résumer à

1 M. DE CERTEAU, L'invention au quotidien, Paris, UGE, 1980, p. 7 (préface). 
de brefs coups de sonde intuitifs dans l'immensité du champ du réel. L'expérience de l'empirie revêt une place déterminante dans la saisie du tour et des alentours du récit.

Est-ce à dire qu'au sein même de l'ORM, et entre celui-ci et la communauté des chercheurs, les débats sont clos à propos des conditions de saisie de l'objet observé ? Certes non. En raison sans doute de la provenance disciplinaire éclatée des membres de l'équipe de recherche, la discussion s'y poursuivra encore longtemps sur la pertinence d'un recours à des analyses exhaustives ou, a contrario, exemplatives afin de pouvoir asseoir la légitimité du sens donné à l'interprétation d'un récit.

Néanmoins, et quoique l'ORM ne revendique pas une identité sociologique, ce qui reviendrait à parjurer son cadre définitionnel originel, il pourrait à ce propos sans doute faire siens ces quelques "antiques" recommandations d'Auguste Comte par lesquels Bourdieu, Chamboredon et Passeron ouvraient leur ouvrage sur le métier de sociologue :

La méthode n'est pas susceptible d'être étudiée séparément des recherches où elle est employée ; ou, du moins, ce n'est là qu'une étude morte, incapable de féconder l'esprit qui s'y livre. Tout ce qu'on peut dire du réel, quand on l'envisage abstraitement, se réduit à des généralités tellement vagues qu'elles ne sauraient avoir aucune influence sur le régime intellectuel. Lorsqu'on a bien établi, en thèse logique, que toutes nos connaissances doivent être fondées sur l'observation, que nous devons procéder tantôt des faits aux principes, et tantôt des principes aux faits, et quelques autres aphorismes semblables, on connaît beaucoup moins nettement la méthode que celui qui a étudié, d'une manière un peu approfondie, une seule science positive, même sans intention philosophique. $(. . .)^{1}$.

\section{Au-delà de la méthode}

Si les propos développés dans le présent ouvrage traitaient bien de méthode, et du positionnement du récit face à ses possibles analyses, est-ce à dire pour autant que le débat s'est confiné à de

1 A. COMTE, Cours de philosophie positive, t.1. Paris, Bachelier, 1830, cité en introduction par P. Bourdieu, J.-C. ChAMBOREDON et J.-C. PASSERON, Le métier de sociologue, Paris, Mouton, 1973, p. 11. 
subtiles gloses sur l'objet de son sujet ? Assurément non. A l'image de ces centaines de milliers de Belges des "marches blanches", dont le mouvement de révolte s'est canalisé autour d'une thématique spécifique alors qu'il s'avérait en fait polysémique, au-delà de la méthode, ou en filigrane à son questionnement, les textes qui précèdent ont aussi voulu s'interroger sur les méthodes pour énoncer autre chose qu'un propos sur la méthode.

Au-delà des méthodes, la véritable question revient à s'interroger sur le sens de la méthode ou, plus précisément, sur sa pertinence visà-vis de son objet. En un mot : des méthodes pour quoi faire ? Pour donner un sens au récit médiatique lui-même, assurément. Mais aussi pour fournir un sens à la portée de son analyse.

Les éléments constitutifs de l'ORM, ses "piliers" portent en euxmêmes ce dépassement de la méthode par le sens. Quelle utilité y aurait-il en effet à justifier la circularité, à plaider la transdisciplinarité, à revendiquer une lecture "transmédiatique" si, au bout de l'analyse, celle-ci se complaisait dans une spéculaire autosatisfaction ou se bornait à entretenir toutes ces composantes dans un seul rapport à l'existence textuelle du récit.

L'ORM le revendique : l'observation de la mise en récit médiatique ne peut s'envisager hors de son contexte social. Son analyste se doit d'utiliser l'étude de la médiation médiatique pour appréhender une réalité sociale. A la fois parce que le contenu médiatique n'existe pas socialement par sa seule existence textuelle ; comme le démontre la structure de l'Observatoire, il n'a de sens social qu'en vertu de son aval et de son amont. Mais aussi parce que l'analyse du récit médiatique telle que l'impose le cadre ricœurien ne revêt une pleine signification que transcendée, c'est-à-dire ramenée à l'échelon du sens de son usage, au niveau de l'"agir et du pâtir".

Comme l'ORM l'énonçait en substance dans l'éditorial d'une de ses "Lettres" intitulé «La recherche comme engagement»1, le chercheur est un acteur social. Il ne peut renier une insertion et une responsabilité spécifique face à la société.

Paraphrasant Alain Touraine, qui évoquait ainsi la fonction de la sociologie, peut-on dès lors mieux conclure ces quelques réflexions qu'en appliquant cette même définition à l'analyse des récits médiatiques et en écrivant ainsi qu' [elle]

1 G. DERÈZE, "La recherche comme engagement", Lettre de l'ORM, $\mathrm{n}^{\circ} 10$, Louvainla-Neuve, octobre 1996, p. 1. 
doit se reconnaître un but et une fonction : contribuer à ce que les membres d'une société s'y comportent le plus possible comme des acteurs et à ce que la société elle-même soit décapée de son ordre, de ses idéologies et de ses rhétoriques, pour apparaître comme un ensemble de systèmes d'action par lesquels, à travers tensions et conflits, un ensemble social agit sur lui-même, sur son organisation et sur son changement ${ }^{1}$.

1 A. Touraine, Pour la sociologie, Paris, Éd. du Seuil, 1974, pp. 237-238. 